\title{
NONSEQUENTIAL SEARCH EQUILIBRIUM WITH SEARCH COST HETEROGENEITY
}

\author{
José Luis Moraga-González \\ Zsolt Sándor \\ Matthijs R. Wildenbeest
}


The Public-Private Sector Research Center is a Research Center based at IESE Business School. Its mission is to develop research that analyses the relationships between the private and public sectors primarily in the following areas: regulation and competition, innovation, regional economy and industrial politics and health economics.

Research results are disseminated through publications, conferences and colloquia. These activities are aimed to foster cooperation between the private sector and public administrations, as well as the exchange of ideas and initiatives.

The sponsors of the SP-SP Center are the following:

- Accenture

- Ajuntament de Barcelona

- Caixa Manresa

- Cambra Oficial de Comerç, Indústria i Navegació de Barcelona

- Departament d' Economia i Finances de la Generalitat de Catalunya

- Departament d' Innovació, Universitats i Empresa de la Generalitat de Catalunya

- Diputació de Barcelona

- Endesa

- FOBSIC

- Fundació AGBAR

- Garrigues

- Institut Català de les Indústries Culturals

- Mediapro

- Microsoft

- Sanofi Aventis

- ATM, FGC y TMB

The contents of this publication reflect the conclusions and findings of the individual authors, and not the opinions of the Center's sponsors. 


\title{
Nonsequential Search Equilibrium with Search Cost Heterogeneity*
}

\author{
José Luis Moraga-González ${ }^{\dagger}$ \\ Zsolt Sándor ${ }^{\ddagger}$ \\ Matthijs R. Wildenbeest ${ }^{\S}$
}

July 2010

\begin{abstract}
We generalize the model of Burdett and Judd (1983) to the case where an arbitrary finite number of firms sells a homogeneous good to buyers who have heterogeneous search costs. We show that a price dispersed symmetric Nash equilibrium always exists. Numerical results show that the behavior of prices with respect to the number of firms hinges upon the shape of the search cost distribution: when search costs are relatively concentrated (dispersed), entry of firms leads to higher (lower) average prices.
\end{abstract}

Keywords: nonsequential search, oligopoly, arbitrary search cost distributions

JEL Classification: D43, C72

\footnotetext{
${ }^{*}$ We are indebted to Allard van der Made, Vladimir Karamychev, Michael Rauh, and specially Paulo K. Monteiro for their useful comments. The paper has also benefited from presentations at a number of seminars and conferences. Financial support from the Netherlands Organization for Scientific Research (NWO) and from Marie Curie Excellence Grant MEXT-CT-2006-042471 is gratefully acknowledged.

${ }^{\dagger}$ ICREA, IESE Business School and University of Groningen, E-mail: j.l.moraga.gonzalez@rug.nl.

${ }^{\ddagger}$ University of Groningen, E-mail: z.sandor@rug.nl.

${ }^{\S}$ Kelley School of Business, Indiana University, E-mail: mwildenb@indiana.edu.
} 


\section{Introduction}

The theory of search has become a toolkit for the understanding of the role of informational imperfections in generating observed market inefficiencies. Burdett and Judd's (1983) model of nonsequential search is one of the seminal contributions. They show that price dispersion can arise as an equilibrium phenomenon in environments where firms and consumers are rational and identical. Burdett and Judd's model of nonsequential search has seen a number of important extensions, including, for example, McAfee's (1995) study of multiproduct firms, Fershtman and Fishman's (1992) study of price dynamics, Acemoglu and Shimer's (2000) study of a general equilibrium labour market, and Janssen and Moraga-González (2004) study of oligopolistic pricing. In labor economics, Burdett and Mortensen's (1998) model has become a canonical framework for explaining wage dispersion and turnover.

This paper generalizes the nonsequential search model studied in Burdett and Judd (1983) to the case in which consumers have heterogeneous search costs. We also work with the finite-number-offirms case, so we can study how price reponsiveness to entry depends on the shape of the search cost distribution. While such an extension has seen applications in empirical work (cf. Hong and Shum, 2006; and Moraga González and Wildenbeest, 2008), the existence and characterization of price dispersed equilibria has not yet been shown. We first demonstrate that firm and consumer optimal behavior can be integrated in such a way that the market equilibrium can be described by an $N$-dimensional nonlinear system of equations. This is useful because of two reasons. First, it provides us with a simple way to simulate the market equilibrium and, second, it enables us to address the existence of equilibrium issue using a fixed point argument. Our main theorem shows that an equilibrium always exists for arbitrary search cost distributions with strictly increasing cdf. In addition, we provide a partial result on uniqueness of equilibrium.

The paper also studies how the number of firms affects equilibrium pricing. We find that the shape of the search cost distribution is relevant. In fact, when consumers have similar search costs, mean prices fall and consumer surplus increases in the number firms. By contrast, if search costs are relatively dispersed across the consumer population, mean prices increase and consumer surplus may fall as the number of firms increases.

The structure of the paper is as follows. In the next section, we present the nonsequential consumer search model studied here. In Section 3 we discuss existence and uniqueness of a price dispersed symmetric equilibrium. In Section 4 we present simulation results illustrating the effects of an increase in the number of firms. All the proofs are placed in the Appendix to ease the reading.

\section{The model}

We examine an oligopolistic version of Burdett and Judd (1983) with consumer search cost heterogeneity. The details of the model follow. $N$ firms produce a good at unit costs that we normalize to zero. ${ }^{1}$ There is a unit mass of buyers. Each consumer inelastically demands one unit of the good and is willing to pay for the good a maximum of $v$. Consumers search for prices nonsequentially and buy from the cheapest store in their sample. Obtaining price quotations, including the first, is costly. Search costs differ across consumers. A buyer's search cost is drawn independently from a common

\footnotetext{
${ }^{1}$ The number of firms can be set equal to infinity in which case $N$ should be interpreted as the maximum number of firms a consumer can obtain price quotations from.
} 
atomless distribution $G(c)$ with support $(0, \infty)$ and positive density $g(c)$ everywhere. A consumer with search cost $c$ sampling $k$ firms incurs a total search cost $k c$.

Firms and buyers play a simultaneous moves game. An individual firm chooses its price taking rivals' prices as well as consumers' search behavior as given. A firm $i$ 's strategy is denoted by a distribution of prices $F_{i}(p)$. Let $F_{-i}(p)$ denote the vector of prices charged by firms other than $i$. The (expected) profit to firm $i$ from charging price $p_{i}$ given rivals' strategies is denoted $\Pi\left(p_{i}, F_{-i}(p)\right)$. Likewise, an individual buyer takes as given firm pricing and decides on his/her optimal search strategy to maximize his/her expected utility. The strategy of a consumer with search cost $c$ is then a number $k$ of prices to sample. Let the fraction of consumers sampling $k$ firms be denoted by $\mu_{k}$. We shall concentrate on symmetric Nash equilibria. A symmetric equilibrium is a distribution of prices $F(p)$ and a collection $\left\{\mu_{0}, \mu_{1}, \ldots, \mu_{N}\right\}$ such that (a) $\Pi_{i}\left(p, F_{-i}(p)\right)$ is equal to a constant $\bar{\Pi}$ for all $p$ in the support of $F(p), \forall i$; (b) $\Pi_{i}\left(p, F_{-i}(p)\right) \leq \bar{\Pi}$ for all $p, \forall i$; (c) a consumer sampling $k$ firms obtains no lower utility than by sampling any other number of firms; and (d) $\sum_{k=0}^{N} \mu_{k}=1$. Let us denote the equilibrium density of prices by $f(p)$, with maximum price $\bar{p}$ and minimum price $\underline{p}$.

\section{Analysis}

We first indicate that, for an equilibrium to exist, there must be some consumers who search just once and others who search more than once.

Proposition 1 If a symmetric equilibrium exists, then $1>\mu_{1}>0$ and $\mu_{k}>0$ for some $k=$ $2,3, \ldots, N$.

The intuition behind this result is simple. Suppose all consumers did search at least twice; then all firms would be subject to price comparisons with rival firms so firm pricing would be competitive. This however is contradictory because then consumers would not be willing to search that much in the first place. Suppose now that no consumer did compare prices; then firms would charge the monopoly price. This is also contradictory because in that case consumers would not be willing to search at all. ${ }^{2}$

We next observe that, given consumer behavior, for an equilibrium to exist it must be the case that firm pricing is characterized by mixed strategies.

Proposition 2 If a symmetric equilibrium exists, $F(p)$ must be atomless with upper bound equal to $v$.

That dispersion must arise is easily understood. If a particular price is chosen with strictly positive probability then a deviant can gain by undercutting such a price. This competition for the pricecomparing consumers cannot drive the price down zero since then a deviant would prefer to raise its price and sell to the consumers who do not compare prices.

We now turn to consumers' search behavior. Expenditure minimization requires a consumer with cost $c$ to continue to draw prices from the price distribution $F(p)$ till the expected gains of searching one more time fall below her search cost. The expected gains from searching $k+1$ prices rather than $k$ prices are given by $E\left[\min \left\{p_{1}, p_{2}, \ldots, p_{k+1}\right\}\right]-E\left[\min \left\{p_{1}, p_{2}, \ldots, p_{k}\right\}\right]$, where $E$ denotes the expectation operator. These gains are strictly positive, decreasing and convergent to zero (see MacMinn, 1980).

\footnotetext{
${ }^{2}$ In the original model of Burdett and Judd (1983) the first price quotation is obtained at no cost and this implies that there always exists an equilibrium where all firms charge the monopoly price.
} 
As a result, a consumer with search cost $c$ will choose to sample $k$ firms provided that the following three inequalities hold:

$$
\begin{array}{r}
v-E\left[\min \left\{p_{1}, p_{2}, \ldots, p_{k}\right\}\right]-k c>0 \\
E\left[\min \left\{p_{1}, p_{2}, \ldots, p_{k-1}\right\}\right]-E\left[\min \left\{p_{1}, p_{2}, \ldots, p_{k}\right\}\right]>c \\
E\left[\min \left\{p_{1}, p_{2}, \ldots, p_{k+1}\right\}\right]-E\left[\min \left\{p_{1}, p_{2}, \ldots, p_{k}\right\}\right]<c
\end{array}
$$

Since the search cost distribution $G(c)$ has support $(0, \infty)$ and positive density everywhere, there exists a consumer indifferent between not searching at all and searching once. Let the search cost of this consumer be denoted $c_{0}$. Then

$$
c_{0}=v-E[p],
$$

since the expected surplus for a consumer who searches one time is $v-E[p]$. Consumers for whom $c \geq c_{0}$ obtain negative surplus if they search. As a result, the share of consumers who do not participate in the market altogether is $\mu_{0}=\int_{c_{0}}^{\infty} d G(c)>0$. Likewise, let $c_{k}$ be the search cost of the consumer indifferent between searching $k$ times and searching $k+1$ times:

$$
c_{k}=E\left[\min \left\{p_{1}, p_{2}, \ldots, p_{k}\right\}\right]-E\left[\min \left\{p_{1}, p_{2}, \ldots, p_{k+1}\right\}\right], k=1,2, \ldots, N-1 .
$$

Consumers for whom $c_{k-1} \leq c \leq c_{k}$ search $k$ times. As a result $\mu_{k}=\int_{c_{k}}^{c_{k-1}} d G(c)>0, k=2,3, \ldots, N$. The following result summarizes:

Proposition 3 Given any atomless price distribution $F(p)$, optimal consumer search behavior is characterized as follows: consumers whose search cost $c \leq c_{N-1}$ search for $N$ prices, consumers whose search cost $c \in\left[c_{k-1}, c_{k}\right]$ search for $k$ prices, $k=1,2, \ldots, N-1$, and consumers whose search cost $c \geq c_{0}$ stay out of the market, where $c_{k}, k=0,1,2, \ldots, N-1$, is given by equations (1) and (2).

Proposition 3 shows that for any given atomless price distribution optimal consumer search leads to a unique grouping of consumers.

We now examine firm pricing behavior. Given consumer search strategies, a firm $i$ charging $p_{i}$ sells to a consumer who compares $k$ prices whenever the price of the other $k-1$ firms is higher than $p_{i}$, which happens with probability $\left(1-F\left(p_{i}\right)\right)^{k-1}$. Therefore the expected profit to firm $i$ from charging price $p_{i}$ when its rivals draw a a price from the cdf $F(p)$ is

$$
\Pi_{i}\left(p_{i} ; F(p)\right)=p_{i}\left(\sum_{k=1}^{N} \frac{k}{N} \mu_{k}\left(1-F\left(p_{i}\right)\right)^{k-1}\right) .
$$

In equilibrium, a firm must be indifferent between charging any price in the support of $F(p)$ and charging the upper bound $\bar{p}$. Thus, any price in the support of $F(p)$ must satisfy $\Pi_{i}\left(p_{i} ; F(p)\right)=$ $\Pi_{i}(\bar{p} ; F(p))$. Since $\Pi_{i}(\bar{p} ; F(p))$ is monotonically increasing in $\bar{p}$, it must be the case that $\bar{p}=v$. As a result, equilibrium requires

$$
\left(p_{i}-r\right)\left[\sum_{k=1}^{N} k \mu_{k}\left(1-F\left(p_{i}\right)\right)^{k-1}\right]=\mu_{1}(v-r) .
$$

Unfortunately, this equation cannot be solved for $F\left(p_{i}\right)$ analytically (except in special cases). However, one can prove existence of an equilibrium price distribution $F\left(p_{i}\right)$. Let us rewrite equation (3) as follows:

$$
\sum_{k=1}^{N} k \mu_{k}\left(1-F\left(p_{i}\right)\right)^{k-1}=\frac{\mu_{1}(v-r)}{\left(p_{i}-r\right)} .
$$


Note that the RHS of equation (4) is positive and does not depend on $F\left(p_{i}\right)$. By contrast, since $F\left(p_{i}\right)$ must take values on $[0,1]$, the LHS of equation (4) is a positive-valued function that decreases in $F\left(p_{i}\right)$ monotonically. At $F\left(p_{i}\right)=0$, the LHS takes on value $\sum_{k=1}^{N} k \mu_{k}$, while at $p_{i}=v$ it takes on value $\mu_{1}$. As a result, for every price $p_{i} \in(\underline{p}, v)$, there is a unique solution to equation (4) satisfying $F\left(p_{i}\right) \in[0,1]$; moreover, the solution $F\left(p_{i} \overline{)}\right.$ is monotonically increasing in $p_{i}$. The following result summarizes these findings.

Proposition 4 Given consumer search behavior $\left\{\mu_{k}\right\}_{k=0}^{N}$, there exists a unique symmetric equilibrium price distribution $F(p)$. In equilibrium firms charge prices randomly chosen from the set $\left[\frac{\mu_{1}(v-r)}{\sum_{k=1}^{N} k \mu_{k}}+r, v\right]$ according to the price distribution defined implicitly by equation (3).

Proposition 4 shows that the equilibrium price distribution is unique for any given grouping of consumers. For the price distribution in Proposition 4 to be an equilibrium of the game, the conjectured grouping of consumers has to be the outcome of optimal consumer search. This requires that the following system of equations holds:

$$
\begin{aligned}
\mu_{k} & =\int_{c_{k}}^{c_{k-1}} d G(c), \text { for all } k=1,2, \ldots, N-1 ; \\
\mu_{N} & =\int_{0}^{c_{N-1}} d G(c),
\end{aligned}
$$

with $\mu_{0}=1-\sum_{k=1}^{N} \mu_{k}$ and where $c_{0}$ and $c_{k}, k=1,2, \ldots, N-1$ are the solutions to

$$
\begin{aligned}
c_{0} & =v-E[p] ; \\
c_{k} & =E\left[\min \left\{p_{1}, p_{2}, \ldots, p_{k}\right\}\right]-E\left[\min \left\{p_{1}, p_{2}, \ldots, p_{k+1}\right\}\right], k=1,2, \ldots, N-1,
\end{aligned}
$$

where the expectation operator is taken over the distribution of prices which solves equation (3).

Using the distributions of the order statistics, and after successively integrating by parts, we can rewrite equations (7) and (8) as follows:

$$
\begin{aligned}
c_{0} & =\int_{\underline{p}}^{v} F(p) d p ; \\
c_{k} & =\int_{\underline{p}}^{v} F(p)(1-F(p))^{k} d p, k=1,2, \ldots, N-1 .
\end{aligned}
$$

$F(p)$ is monotonically increasing in $p$ so we can use equation (3) to find its inverse:

$$
p(z)=\frac{\mu_{1}(v-r)}{\sum_{k=1}^{N} k \mu_{k}(1-z)^{k-1}}+r .
$$

Using this inverse function, integration by parts and the change of variables $z=F(p)$ in equations (9) and (10) yields:

$$
\begin{aligned}
& c_{0}=v-\int_{0}^{1} p(z) d z \\
& c_{k}=\int_{0}^{1} p(z)[(k+1) z-1](1-z)^{k-1} d z, k=1,2, \ldots, N-1 .
\end{aligned}
$$

Therefore we can state that: 
Proposition 5 If a symmetric equilibrium of the game exists then consumers search according to Proposition 3, firms set prices according to Proposition 4, and the series of critical cutoff points $\left\{c_{k}\right\}_{k=0}^{N-1}$ is given by the solution to the system of equations:

$$
\begin{aligned}
& c_{0}=(v-r)\left(1-\int_{0}^{1} \frac{G\left(c_{0}\right)-G\left(c_{1}\right)}{\sum_{k=1}^{N} k\left[G\left(c_{k-1}\right)-G\left(c_{k}\right)\right] u^{k-1}} d u\right) ; \\
& c_{k}=(v-r) \int_{0}^{1} \frac{\left[G\left(c_{0}\right)-G\left(c_{1}\right)\right]\left[k u^{k-1}-(k+1) u^{k}\right]}{\sum_{k=1}^{N} k\left[G\left(c_{k-1}\right)-G\left(c_{k}\right)\right] u^{k-1}} d u, k=1,2, \ldots, N-1 .
\end{aligned}
$$

This result is useful for two reasons. First, it provides a straightforward way to compute and simulate the market equilibrium. For fixed $v, r$ and $G(c)$, the system of equations (14)-(15) can be solved numerically. If a solution exists, then the consumer equilibrium is given by equations (5)-(6) and the price distribution follows readily from equation (11). Secondly, this result enables us to address the existence and uniqueness of equilibrium issues, which are the subject of our next statement.

Theorem 1 For any consumer valuation $v$ and firm marginal cost $r$ such that $v>r \geq 0$ and for any search cost distribution function $G(c)$ with support $(0, \infty)$ such that either $g(0)>0$ or $g(0)=0$ and $g^{\prime}(0)>0$, an equilibrium exists in a market with an arbitrary number of firms $N$. Moreover, when $N=2$ and $g^{\prime}(\cdot) \simeq 0$, there exists a unique equilibrium.

The proof of this result, which is in the Appendix, builds on Brouwer's fixed point theorem. To apply the theorem, we first construct an auxiliary mapping and show that a market equilibrium is given by a fixed point of such mapping. A difficulty we encounter in applying Brouwer's fixed point theorem directly is that the auxiliary mapping happens to be discontinuous at zero. This would not be a problem if we could bound the domain of definition of the auxiliary mapping. However, it is not possible to find a bound of the domain of definition that is appropriate for arbitrary search cost distributions. Because of this, we modify the auxiliary mapping in the neighborhood of 0 and apply Brouwer's fixed point theorem to the modified auxiliary mapping.

Theorem 1 also establishes uniqueness of equilibrium when the market is operated by two firms and the search cost distribution is uniform. General results on uniqueness prove to be very difficult because we cannot compute the equilibrium explicitly. However, simulations of the model for different parameters and search cost distributions suggest the uniqueness result is more general.

\section{Price equilibrium and the number of firms}

In this section we illustrate the importance of the shape of the search cost distribution for pricing. In particular we focus on how entry affects pricing for different search cost distributions. ${ }^{3}$ The price and welfare effects of entry in our model are difficult to derive analytically since the equilibrium price distribution cannot be obtained in closed-form. We then proceed by solving the model numerically.

Consider a market where consumer valuations are identical and let $v=100$; in addition, assume that the first price quotation is obtained at no cost. These two assumptions together imply that all

\footnotetext{
${ }^{3}$ Janssen and Moraga-González (2004) study the effects of entry in a model with a two-point search cost distribution that includes an atom of shoppers.
} 
consumers buy in equilibrium. This is convenient since it allows us to isolate the single mechanism through which entry influences the aggregate outcome: the amount of search. The firms' marginal cost $r$ is set equal to 50 .

Let us assume that search costs follow a log-normal distribution, with parameters $\left(\nu_{c}, \sigma_{c}\right)$. In what follows, we fix the mean search cost to 50 and compare how the market works for two different levels of search cost dispersion. In particular, we focus on the effects of entry on prices and surplus and study how these effects depend on the amount of search cost dispersion. We start with a market where search cost dispersion is relatively low. For this we set $\left(\nu_{c}, \sigma_{c}\right)=(2.63,1.6) .{ }^{4}$ Given the other data, we solve for the equilibrium of the model for different number of firms. The results are reported in Table 1.

\begin{tabular}{ccccccccccccc}
\hline & $N=2$ & $N=3$ & $N=4$ & $N=5$ & $N=6$ & $N=7$ & $N=8$ & $N=9$ & $N=10$ & $N=11$ & $N=12$ & $N=13$ \\
\hline$\mu_{1}$ & 1.00 & 0.86 & 0.81 & 0.79 & 0.78 & 0.78 & 0.78 & 0.78 & 0.77 & 0.77 & 0.77 & 0.77 \\
$\mu_{2}$ & 0.00 & 0.07 & 0.09 & 0.09 & 0.09 & 0.09 & 0.09 & 0.09 & 0.09 & 0.09 & 0.09 & 0.09 \\
$\mu_{3}$ & - & 0.07 & 0.04 & 0.05 & 0.05 & 0.05 & 0.05 & 0.05 & 0.05 & 0.05 & 0.05 & 0.05 \\
$\mu_{4}$ & - & - & 0.06 & 0.03 & 0.03 & 0.03 & 0.03 & 0.03 & 0.03 & 0.03 & 0.03 & 0.03 \\
$\mu_{5}$ & - & - & - & 0.05 & 0.02 & 0.02 & 0.02 & 0.02 & 0.02 & 0.02 & 0.02 & 0.02 \\
$\mu_{6}$ & - & - & - & - & 0.04 & 0.01 & 0.01 & 0.01 & 0.01 & 0.01 & 0.01 & 0.01 \\
$\mu_{7}$ & - & - & - & - & - & 0.03 & 0.01 & 0.01 & 0.01 & 0.01 & 0.01 & 0.01 \\
$\mu_{8}$ & - & - & - & - & - & - & 0.02 & 0.01 & 0.01 & 0.01 & 0.01 & 0.01 \\
$\mu_{9}$ & - & - & - & - & - & - & - & 0.02 & 0.00 & 0.00 & 0.00 \\
$\mu_{10}$ & - & - & - & - & - & - & - & - & 0.01 & 0.00 & 0.00 \\
$\mu_{11}$ & - & - & - & - & - & - & - & - & - & 0.00 \\
$\mu_{12}$ & - & - & - & - & - & - & - & - & - & - & 0.01 & 0.00 \\
$\mu_{13}$ & - & - & - & - & - & - & - & - & - & 0.00 \\
\hline
\end{tabular}

Table 1: Equilibrium search intensities for $\left(\nu_{c}, \sigma_{c}\right)=(2.63,1.6)$ (mean is 50; CV is 3.45)

Table 1 shows how consumer search intensities change as we increase the number of firms. Remarkably, in this market a large majority of the consumers searches only once. For example, when there are just two firms in the market the fraction of consumers who do not compare prices is almost $100 \%$. This number remains high but decreases as we increase the number of firms. A second important feature is that very few consumers make an exhaustive search in the market; in fact for example if there are 10 firms in the industry about $94 \%$ of the consumers searches for a maximum of 4 firms.

\section{$<$ insert figure 1 about here $>$}

The fact that most consumers do not compare prices is reflected in equilibrium prices. Figure 1(a) shows how mean prices change with the number of firms. The average price under duopoly is very high and it decreases as the number of firms rises. The decrease of the mean price is due to the fact that the share of consumers comparing two or three prices increases in the number of firms. The average price is what is important for consumers who do not exercise price comparisons so consumers benefit from the resulting average price decreases. These gains are also reflected in that consumer surplus, plotted in Figure 1(b), increases in $N$. Figures 1(c) and 1(d) show the behavior of aggregate profits and social welfare. The welfare result is perhaps surprising and it deserves an explanation. Note from Table 1 that the amount of search increases in $N$ and that, since search costs are wasteful, more search generates a welfare loss. In sum, entry in this case of low search cost dispersion would lead to lower average prices, higher consumer surplus, lower industry profits and higher welfare.

\footnotetext{
${ }^{4}$ Mean search cost is equal to $\mathrm{e}^{\nu+\sigma^{2} / 2} \simeq 50$ and standard deviation $\sqrt{\left(\mathrm{e}^{\sigma^{2}}-1\right) \mathrm{e}^{2 \nu+\sigma^{2}}} \simeq 172.74$.
} 
The situation is quite different when search costs are much more dispersed, holding everything else equal. Let us set $\left(\nu_{c}, \sigma_{c}\right)=(0.79,2.5)$, which implies the new search cost distribution is a meanpreserving spread of the previous one. The new equilibrium search intensities are reported in Table 2. What is different in this case of high search cost dispersion is that a great deal of consumers conduct an exhaustive search; as before, the extent of price comparison in the market increases as the number of firms rises.

\begin{tabular}{|c|c|c|c|c|c|c|c|c|c|c|c|c|}
\hline & $N=2$ & $N=3$ & $N=4$ & $N=5$ & $N=6$ & $N=7$ & $N=8$ & $N=9$ & $N=10$ & $N=11$ & $N=12$ & $N=13$ \\
\hline$\mu_{1}$ & 0.37 & 0.32 & 0.30 & 0.30 & 0.29 & 0.29 & 0.29 & 0.29 & 0.29 & 0.29 & 0.29 & 0.29 \\
\hline$\mu_{2}$ & 0.63 & 0.14 & 0.12 & 0.11 & 0.11 & 0.10 & 0.10 & 0.10 & 0.10 & 0.10 & 0.10 & 0.10 \\
\hline$\mu_{3}$ & - & 0.54 & 0.10 & 0.10 & 0.09 & 0.09 & 0.08 & 0.08 & 0.08 & 0.08 & 0.08 & 0.08 \\
\hline$\mu_{4}$ & - & - & 0.47 & 0.08 & 0.08 & 0.07 & 0.07 & 0.07 & 0.07 & 0.07 & 0.07 & 0.07 \\
\hline$\mu_{5}$ & - & - & - & 0.42 & 0.06 & 0.06 & 0.06 & 0.06 & 0.06 & 0.06 & 0.06 & 0.06 \\
\hline$\mu_{6}$ & - & - & - & - & 0.37 & 0.05 & 0.05 & 0.05 & 0.05 & 0.05 & 0.05 & 0.05 \\
\hline$\mu_{7}$ & - & - & - & - & - & 0.33 & 0.04 & 0.04 & 0.04 & 0.04 & 0.04 & 0.04 \\
\hline$\mu_{8}$ & - & - & - & - & - & - & 0.30 & 0.04 & 0.04 & 0.04 & 0.04 & 0.03 \\
\hline$\mu_{9}$ & - & - & - & - & - & - & - & 0.27 & 0.03 & 0.03 & 0.03 & 0.03 \\
\hline$\mu_{10}$ & - & - & - & - & - & - & - & - & 0.25 & 0.03 & 0.03 & 0.03 \\
\hline$\mu_{11}$ & - & - & - & - & - & - & - & - & - & 0.22 & 0.02 & 0.02 \\
\hline$\mu_{12}$ & - & - & - & - & - & - & - & - & - & - & 0.21 & 0.02 \\
\hline$\mu_{13}$ & - & - & - & - & - & - & - & - & - & - & - & 0.19 \\
\hline
\end{tabular}

Table 2: Equilibrium search intensities for $\left(\nu_{c}, \sigma_{c}\right)=(0.79,2.5)$ (mean is 50; CV is 22.73)

Figure 2 plots the equilibrium mean price against the number of competitors in the industry. Under duopoly, the average price is relatively low compared to the previous case. What is remarkably different is that the mean price increases as more firms enter the industry. Moreover, we see that consumer surplus can decrease and profits increase as we the number of competitors goes up. The crucial distinction between this case and the previous one is the equilibrium consumer search intensity. Table 2 shows that most of the consumers (more than 63\%) exercise price comparisons in this case while Table 1 showed the opposite evidence. Consumers who conduct an exhaustive search in the market become disproportionately less attractive for a firm as more competitors are around. This effect, which leads to higher prices, has here a dominating influence and results in lower consumer surplus and greater industry profits. Welfare is again decreasing in $N$ due to the rise of actually incurred search costs.

$$
<\text { insert figure } 2 \text { about here }>
$$

In summary, this section shows that entry can lead to an increase or to a decrease in average prices and that the direction of the effect depends on the extent of search frictions in the market. 


\section{Appendix}

Proof of Proposition 1. First, suppose, on the contrary, that $\mu_{1}=0$. Then we have two possibilities: $(i)$ either $\mu_{0}=1$ in which case the market does not open, or $(i i) \mu_{k}>0$ for some $k=2,3, \ldots, N$ in which case all firms would charge a price equal to the marginal cost $r$. But if this were so, consumers would gain by deviating and searching less. Second, suppose, on the contrary, that $\mu_{1}=1$. Then firms prices would be equal to the monopoly price $v$. But if this were so then consumers would gain by deviating and exiting the market. Finally, suppose, on the contrary, that $1>\mu_{1}>0$ and that $\mu_{k}=0$ for all $k=2,3, \ldots, N$. Then $\mu_{0}+\mu_{1}=1$ and the argument applied before would hold here too; as a result, there must be some $k \geq 2$ for which $\mu_{k}>0$.

Proof of Proposition 2. Suppose, on the contrary, that firms did charge a price $\hat{p} \in(r, v]$ with strictly positive probability in equilibrium. Consider a firm $i$ charging $\hat{p}$. The probability that $\hat{p}$ is the only price in the market is strictly positive. This occurs when all other firms are charging $\hat{p}$. From Proposition 1 we know that in equilibrium there exists some $\hat{k} \geq 2$ for which $\mu_{\hat{k}}>0$. Consider the fraction of consumers sampling $\hat{k}$ firms. The probability that these consumers are sampling firm $i$ is strictly positive; as a result, firm $i$ would gain by deviating and charging $\hat{p}-\varepsilon$ since in that case the firm would attract all consumers in $\mu_{\hat{k}}$ who happened to sample firm $i$. This deviation would give firm $i$ a discrete increase in its profits and thus rules out all atoms in the set $(r, v]$. It remains to be proven that an atom at the marginal cost $r$ cannot be part of an equilibrium either. Consider a firm charging $r$. From Proposition 1 we know that $1>\mu_{1}>0$. As a result, this firm would serve a fraction of consumers at least as large as $\mu_{1} / N$ but obtain zero profits. This implies that the firm would have an incentive to deviate by increasing its price. We now prove that the upper bound of $F(p)$ must be equal to $v$. Suppose not and consider a firm charging an upper bound $\bar{p}<v$. Since this firm would not sell to any consumer who compares prices, its payoff would simply be equal to $(\bar{p}-r) \mu_{1} / N$, which is strictly increasing in $\bar{p}$; as a result the firm would gain by deviating and charging $v$.

Proof of Theorem 1. Let $\theta:=v-r$ and consider the change of variables $x_{k}:=G\left(c_{k}\right)$. Then we can rewrite the equations describing the equilibrium (14)-(15) as

$$
\begin{aligned}
& x_{0}=G\left(\theta-\theta \int_{0}^{1} \frac{x_{0}-x_{1}}{\sum_{h=1}^{N} h\left(x_{h-1}-x_{h}\right) u^{h-1}} d u\right) \\
& x_{k}=G\left(\theta \int_{0}^{1} \frac{x_{0}-x_{1}}{\sum_{h=1}^{N} h\left(x_{h-1}-x_{h}\right) u^{h-1}}\left[k u^{k-1}-(k+1) u^{k}\right] d u\right), k=1,2, \ldots, N-1, \quad\left(x_{N}=0\right) .
\end{aligned}
$$

Since $x_{0}=G\left(c_{0}\right)>0$ in any interesting market equilibrium, we can define $y_{k}=\frac{x_{k}}{x_{0}}$. Then the solution of this system will be

$$
\begin{aligned}
& x_{0}=G\left(\theta-\theta \int_{0}^{1} \frac{1-y_{1}}{1-y_{1}+\sum_{h=2}^{N} h\left(y_{h-1}-y_{h}\right) u^{h-1}} d u\right), \\
& x_{1}=x_{0} y_{1}, \ldots, x_{N-1}=x_{0} y_{N-1},
\end{aligned}
$$

if $y=\left(y_{1}, y_{2}, \ldots, y_{N-1}\right)$ is the solution of the following system of equations:

$$
y_{k}=\frac{G\left(\theta \int_{0}^{1} \frac{\left(1-y_{1}\right)\left[k u^{k-1}-(k+1) u^{k}\right]}{1-y_{1}+\sum_{h=2}^{N} h\left(y_{h-1}-y_{h}\right) u^{h-1}} d u\right)}{G\left(\theta-\theta \int_{0}^{1} \frac{1-y_{1}}{1-y_{1}+\sum_{h=2}^{N} h\left(y_{h-1}-y_{h}\right) u^{h-1}} d u\right)}, k=1,2, \ldots, N-1, \quad\left(y_{N}=0\right) .
$$


We are looking for a solution of this latter system in $[0,1]^{N-1}$ for which $y_{1} \geq y_{2} \geq \ldots \geq y_{N-1}$. For this purpose, we define the set $Y=\left\{\left(y_{1}, y_{2}, \ldots, y_{N-1}\right) \in[0,1]^{N-1}: y_{1} \geq y_{2} \geq \ldots \geq y_{N-1}\right\}$. Likewise, define the function $H=\left(H_{1}, \ldots, H_{N-1}\right): Y \backslash\{0\} \rightarrow \mathbb{R}^{N-1}$ with

$$
H_{k}(y)=\frac{G\left(\theta \int_{0}^{1} \frac{\left(1-y_{1}\right)\left[k u^{k-1}-(k+1) u^{k}\right]}{1-y_{1}+\sum_{h=2}^{N} h\left(y_{h-1}-y_{h}\right) u^{h-1}} d u\right)}{G\left(\theta-\theta \int_{0}^{1} \frac{1-y_{1}}{1-y_{1}+\sum_{h=2}^{N} h\left(y_{h-1}-y_{h}\right) u^{h-1}} d u\right)}, \quad k=1,2, \ldots, N-1, \quad\left(y_{N}=0\right) .
$$

Then the solution of the system (16) is a fixed point of $H$. In what follows we apply Brouwer's theorem to show that the function $H$ has a fixed point.

First we show that the function $H$ takes values in the set $Y$. This is intuitively clear based on the properties of the model since by appropriate transformations it is equivalent to the inequalities $c_{0} \geq c_{1} \geq \ldots \geq c_{N-1}$. Here we provide a direct proof.

Lemma 1 The function $H(\cdot)$ takes values in $Y$.

Proof. Take an arbitrary $y \in Y \backslash\{0\}$. We need to prove that $0 \leq H_{k}(y) \leq 1$ for all $k=1,2, \ldots, N-1$ and $H_{k}(y) \leq H_{k-1}(y)$ for all $k=2, \ldots, N-1$. The inequality $0 \leq H_{k}(y)$ follows straightforwardly from the nonnegativity of $G$. In order to prove $H_{k}(y) \leq 1$ and $H_{k}(y) \leq H_{k-1}(y)$ we use integration by parts. First we observe that

$$
\int_{0}^{1} \frac{1-y_{1}}{1-y_{1}+\sum_{h=2}^{N} h\left(y_{h-1}-y_{h}\right) u^{h-1}} d u=\int_{0}^{1} \frac{1-y_{1}}{1-y_{1}+\sum_{h=2}^{N} h\left(y_{h-1}-y_{h}\right)(1-u)^{h-1}} d u .
$$

By integration by parts

$$
\begin{aligned}
& \int_{0}^{1} \frac{1-y_{1}}{1-y_{1}+\sum_{h=2}^{N} h\left(y_{h-1}-y_{h}\right)(1-u)^{h-1}} d u \\
& =1-\int_{0}^{1} \frac{\left(1-y_{1}\right) u\left[\sum_{h=2}^{N} h(h-1)\left(y_{h-1}-y_{h}\right)(1-u)^{h-2}\right]}{\left(1-y_{1}+\sum_{h=2}^{N} h\left(y_{h-1}-y_{h}\right)(1-u)^{h-1}\right)^{2}} d u .
\end{aligned}
$$

So the argument of $G$ in the denominator is proportional to

$$
\begin{aligned}
& 1-\int_{0}^{1} \frac{1-y_{1}}{1-y_{1}+\sum_{h=2}^{N} h\left(y_{h-1}-y_{h}\right) u^{h-1}} d u \\
& =\int_{0}^{1} \frac{\left(1-y_{1}\right) u\left[\sum_{h=2}^{N} h(h-1)\left(y_{h-1}-y_{h}\right) u^{h-2}\right]}{\left(1-y_{1}+\sum_{h=2}^{N} h\left(y_{h-1}-y_{h}\right) u^{h-1}\right)^{2}} d u .
\end{aligned}
$$

The argument of $G$ in the numerator of $H_{k}(\cdot)$ is proportional to

$$
\begin{aligned}
& \int_{0}^{1} \frac{\left(1-y_{1}\right)\left[k u^{k-1}-(k+1) u^{k}\right]}{1-y_{1}+\sum_{h=2}^{N} h\left(y_{h-1}-y_{h}\right) u^{h-1}} d u \\
& =\int_{0}^{1} \frac{1-y_{1}}{1-y_{1}+\sum_{h=2}^{N} h\left(y_{h-1}-y_{h}\right) u^{h-1}} d\left(u^{k}-u^{k+1}\right) \\
& =\int_{0}^{1} \frac{\left(1-y_{1}\right) u^{k}(1-u)\left[\sum_{h=2}^{N} h(h-1)\left(y_{h-1}-y_{h}\right) u^{h-2}\right]}{\left(1-y_{1}+\sum_{h=2}^{N} h\left(y_{h-1}-y_{h}\right) u^{h-1}\right)^{2}} d u .
\end{aligned}
$$


The inequality $H_{k}(y) \leq 1$ follows from the fact that $u \geq u^{k}(1-u)$ while the inequalities $H_{k}(y) \leq$ $H_{k-1}(y), k=2,3, \ldots, N-1$ follow because all terms in the expressions of the integrals are nonnegative and $u^{k}$ is decreasing in $k$.

We now apply Brouwer's fixed point theorem to prove a fixed point of $H$ exists. Since the denominator of $H_{k}$ is 0 for $y=0$, we need to modify the function $H$ in the neighborhood of 0 . We do this in three steps: $(i)$ We first prove that the limit inferior of $H$ when $y \rightarrow 0$ is strictly positive (Proposition 6). (ii) We then construct a neighborhood $V$ of 0 such that $H$ is continuously extendable from $Y \backslash V$ to $Y$ such that the extended function has no fixed point in $V$ (Lemma 3, Lemma 4). (iii) Finally, we apply Brouwer's fixed point theorem to the extended function to establish the existence of a solution of the system (16).

We start by showing that the limit inferior of $H$ is strictly positive. Since $H_{k}(y) \leq H_{k-1}(y)$, $k=2,3, \ldots, N-1$, is is sufficient to study the limit inferior of $H_{1}$.

Proposition $6 \liminf _{\substack{y \in Y \\ y \in Y}} H_{1}(y) \geq \begin{cases}\frac{1}{3} & \text { if } g(0)>0, \\ \frac{1}{9} & \text { if } g(0)=0 \text { and } g^{\prime}(0)>0 .\end{cases}$

Proof. By definition $\liminf _{\substack{y \rightarrow 0 \\ y \in Y}} H_{1}(y)=\lim _{\varepsilon \rightarrow 0} \inf \left\{H_{1}(y): y \in Y \cap B(0, \varepsilon) \backslash\{0\}\right\}$, where $B(0, \varepsilon)=$ $\left\{x \in \mathbb{R}^{N-1}:\|x\|<\varepsilon\right\}$. By Lemma 2 below there exists an $\varepsilon>0$ such that $H_{1}(y)$ is increasing in $y_{k}$ for $k=2, \ldots, N-1$ on $Y \cap B(0, \varepsilon) \backslash\{0\}$. This implies that for any $y \in Y \cap B(0, \varepsilon) \backslash\{0\}$ such that $y_{1}>0$

$$
\begin{aligned}
H_{1}\left(y_{1}, y_{2}, \ldots, y_{N-1}\right) & \geq H_{1}\left(y_{1}, y_{2}, \ldots, y_{N-2}, 0\right) \geq H_{1}\left(y_{1}, y_{2}, \ldots, 0,0\right) \geq \ldots \geq H_{1}\left(y_{1}, 0, \ldots, 0\right) \\
& =\frac{G\left(\theta \int_{0}^{1} \frac{\left(1-y_{1}\right)(1-2 u)}{1-y_{1}+2 y_{1} u} d u\right)}{G\left(\theta-\theta \int_{0}^{1} \frac{1-y_{1}}{1-y_{1}+2 y_{1} u} d u\right)} .
\end{aligned}
$$

Therefore,

$$
\lim \inf _{\substack{y \rightarrow 0 \\ y \in Y}} H_{1}(y) \geq \lim _{\varepsilon \rightarrow 0} \inf \left\{\frac{G\left(\theta \int_{0}^{1} \frac{\left(1-y_{1}\right)(1-2 u)}{1-y_{1}+2 y_{1} u} d u\right)}{G\left(\theta-\theta \int_{0}^{1} \frac{1-y_{1}}{1-y_{1}+2 y_{1} u} d u\right)}: 0<y_{1}<\varepsilon\right\} .
$$

The limit on the right hand side is by definition the limit inferior of $\frac{G\left(\theta \int_{0}^{1} \frac{\left(1-y_{1}\right)(1-2 u)}{1-y_{1}+2 y_{1} u} d u\right)}{G\left(\theta-\theta \int_{0}^{1} \frac{1-y_{1}}{1-y_{1}+2 y_{1} u} d u\right)}$ when $y_{1} \rightarrow 0, y_{1}>0$. We show that this limit inferior is just equal to the limit, due to the fact that the limit exists. Indeed, we can apply the l'Hôpital rule to obtain

$$
\lim _{\substack{y_{1} \rightarrow 0 \\ y_{1}>0}} \frac{G\left(\theta \int_{0}^{1} \frac{\left(1-y_{1}\right)(1-2 u)}{1-y_{1}+2 y_{1} u} d u\right)}{G\left(\theta-\theta \int_{0}^{1} \frac{1-y_{1}}{1-y_{1}+2 y_{1} u} d u\right)}=\lim _{\substack{y_{1} \rightarrow 0 \\ y_{1}>0}}-\frac{g\left(\theta \int_{0}^{1} \frac{\left(1-y_{1}\right)(1-2 u)}{1-y_{1}+2 y_{1} u} d u\right) \int_{0}^{1} \frac{u(1-2 u)}{\left(1-y_{1}+2 y_{1} u\right)^{2}} d u}{g\left(\theta-\theta \int_{0}^{1} \frac{1-y_{1}}{1-y_{1}+2 y_{1} u} d u\right) \int_{0}^{1} \frac{u}{\left(1-y_{1}+2 y_{1} u\right)^{2}} d u} .
$$

If $g(0)>0$ then this limit is further equal to

$$
-\frac{g\left(\theta \int_{0}^{1}(1-2 u) d u\right) \int_{0}^{1} u(1-2 u) d u}{g\left(\theta-\theta \int_{0}^{1} d u\right) \int_{0}^{1} u d u}=-\frac{g(0) \int_{0}^{1} u(1-2 u) d u}{g(0) \int_{0}^{1} u d u}=-\frac{\int_{0}^{1} u(1-2 u) d u}{\int_{0}^{1} u d u}=\frac{1}{3} .
$$


If $g(0)=0$ and $g^{\prime}(0)>0$ then the limit (17) is equal to the limit of

$$
\begin{aligned}
& -\frac{g^{\prime}\left(\theta \int_{0}^{1} \frac{\left(1-y_{1}\right)(1-2 u) d u}{1-y_{1}+2 y_{1} u}\right) \theta \int_{0}^{1} \frac{-2 u(1-2 u) d u}{\left(1-y_{1}+2 y_{1} u\right)^{2}} \int_{0}^{1} \frac{u(1-2 u) d u}{\left(1-y_{1}+2 y_{1} u\right)^{2}}+g\left(\theta \int_{0}^{1} \frac{\left(1-y_{1}\right)(1-2 u) d u}{1-y_{1}+2 y_{1} u}\right) \int_{0}^{1} \frac{2 u(1-2 u)^{2} d u}{\left(2 u y_{1}-y_{1}+1\right)^{3}}}{g^{\prime}\left(\theta-\theta \int_{0}^{1} \frac{\left(1-y_{1}\right) d u}{1-y_{1}+2 y_{1} u}\right) \int_{0}^{1} \frac{(-\theta)(-2 u) d u}{\left(1-y_{1}+2 y_{1} u\right)^{2}} \int_{0}^{1} \frac{u d u}{\left(1-y_{1}+2 y_{1} u\right)^{2}}+g\left(\theta-\theta \int_{0}^{1} \frac{\left(1-y_{1}\right) d u}{1-y_{1}+2 y_{1} u}\right) \int_{0}^{1} \frac{2 u(1-2 u) d u}{\left(2 u y_{1}-y_{1}+1\right)^{3}}} \\
= & -\frac{g^{\prime}(0) \theta \int_{0}^{1}(-2 u)(1-2 u) d u \int_{0}^{1} u(1-2 u) d u+g(0) \int_{0}^{1} 2 u(1-2 u)^{2} d u}{g^{\prime}(0)(-\theta) \int_{0}^{1}(-2 u) d u \int_{0}^{1} u d u+g(0) \int_{0}^{1} 2 u(1-2 u) d u} \\
= & \frac{\int_{0}^{1}(-2 u)(1-2 u) d u \int_{0}^{1} u(1-2 u) d u}{\int_{0}^{1}(-2 u) d u \int_{0}^{1} u d u}=\frac{1}{9} .
\end{aligned}
$$

Lemma 2 There exists an $\varepsilon>0$ such that $H_{1}(y)$ is increasing in $y_{k}$ for $k=2, \ldots, N-1$ on $Y \cap B(0, \varepsilon) \backslash\{0\}$.

Proof. For simplicity of notation we use

$$
H_{1}(y)=\frac{U(y)}{D(y)},
$$

where $U, D: Y \rightarrow \mathbb{R}$

$$
\begin{aligned}
& U(y)=G\left(\theta \int_{0}^{1} \frac{\left(1-y_{1}\right)(1-2 u)}{1-y_{1}+\sum_{h=2}^{N} h\left(y_{h-1}-y_{h}\right) u^{h-1}} d u\right), \\
& D(y)=G\left(\theta-\theta \int_{0}^{1} \frac{1-y_{1}}{1-y_{1}+\sum_{h=2}^{N} h\left(y_{h-1}-y_{h}\right) u^{h-1}} d u\right) .
\end{aligned}
$$

The partial derivatives of $U$ and $D$ with respect to $y_{k}$ for some $k \in\{2, \ldots, N-1\}$ are

$$
\begin{aligned}
\frac{\partial U}{\partial y_{k}} & =g\left(\theta \int_{0}^{1} \frac{\left(1-y_{1}\right)(1-2 u)}{1-y_{1}+\sum_{h=2}^{N} h\left(y_{h-1}-y_{h}\right) u^{h-1}} d u\right) \theta I_{U}(y), \\
\frac{\partial D}{\partial y_{k}} & =g\left(\theta-\theta \int_{0}^{1} \frac{1-y_{1}}{1-y_{1}+\sum_{h=2}^{N} h\left(y_{h-1}-y_{h}\right) u^{h-1}} d u\right)(-\theta) I_{D}(y),
\end{aligned}
$$

where

$$
\begin{aligned}
& I_{U}(y)=\int_{0}^{1} \frac{\left(1-y_{1}\right)(1-2 u)\left[k u^{k-1}-(k+1) u^{k}\right]}{\left(1-y_{1}+\sum_{h=2}^{N} h\left(y_{h-1}-y_{h}\right) u^{h-1}\right)^{2}} d u, \\
& I_{D}(y)=\int_{0}^{1} \frac{\left(1-y_{1}\right)\left[k u^{k-1}-(k+1) u^{k}\right]}{\left(1-y_{1}+\sum_{h=2}^{N} h\left(y_{h-1}-y_{h}\right) u^{h-1}\right)^{2}} d u .
\end{aligned}
$$

By integration by parts

$$
I_{D}(y)=2 \int_{0}^{1}\left(1-y_{1}\right)\left(u^{k}-u^{k+1}\right) \frac{\sum_{h=2}^{N} h(h-1)\left(y_{h-1}-y_{h}\right) u^{h-2}}{\left(1-y_{1}+\sum_{h=2}^{N} h\left(y_{h-1}-y_{h}\right) u^{h-1}\right)^{3}} d u .
$$


Now, $I_{D} \geq 0$ for any $y \in Y$ because all terms in the integral are nonnegative. Therefore $\frac{\partial D}{\partial y_{k}} \leq 0$ for any $y \in Y$, which implies that $D$ is decreasing in $y_{k}$ at any point $y \in Y$.

Regarding the integral $I_{U}$ we note that

$$
I_{U}(0)=\int_{0}^{1}(1-2 u)\left[k u^{k-1}-(k+1) u^{k}\right] d u=\frac{2}{(k+1)(k+2)}>0 .
$$

So for each $k$ there is an $\varepsilon_{k}>0$ such that $I_{U}(y) \geq 0$ for any $y \in Y \cap B\left(0, \varepsilon_{k}\right)$; so for $\varepsilon=$ $\min \left\{\varepsilon_{2}, \ldots, \varepsilon_{N-1}\right\}$ it holds that $I_{U}(y) \geq 0$ for any $y \in Y \cap B(0, \varepsilon)$. Therefore $\frac{\partial U}{\partial y_{k}} \geq 0$ for any $y \in Y \cap B(0, \varepsilon)$ and $k=2, \ldots, N-1$. This implies that $U$ is increasing in $y_{k}$ for any $y \in Y \cap B(0, \varepsilon)$. This establishes that $H_{1}(y)$ is increasing in $y_{k}$ for any $y \in Y \cap B(0, \varepsilon) \backslash\{0\}$.

So we have established that the limit inferior of $H_{1}(y)$ when $y \rightarrow 0$ is strictly positive. Then the following statement establishes that there is an $\varepsilon>0$ such that the set $Y \cap[0, \varepsilon]^{N-1}$ can take the role of the neighborhood $V$ mentioned above.

Lemma 3 Let $H: Y \backslash\{0\} \rightarrow \mathbb{R}^{N-1}$ be a continuous function such that $\liminf _{\substack{y \rightarrow 0 \\ y \in Y}} H_{1}(y) \geq a>0$. Then there exists $\varepsilon>0$ such that $H_{1}(y)>\varepsilon$ for any $y=\left(y_{1}, y_{2}, \ldots, y_{N-1}\right) \in Y \backslash\{0\}$ with $y_{1} \leq \varepsilon$.

Proof. Condition $\liminf _{\substack{y \rightarrow 0 \\ y \in Y}} H_{1}(y) \geq a>0$ implies that for any $\delta>0$ there exists $\varepsilon_{\delta}>0$ such that $H_{1}(y)>a-\delta$ for any $y=\left(y_{1}, y_{2}, \ldots, y_{N-1}\right) \in Y \backslash\{0\}$ with $y_{1} \leq \varepsilon_{\delta}$. Take $\delta_{1}>0$ such that $a-\delta_{1}>0$. Then there exists $\varepsilon_{1}>0$ such that $H_{1}(y)>a-\delta_{1}$ for any $y=\left(y_{1}, y_{2}, \ldots, y_{N-1}\right) \in Y \backslash\{0\}$ with $y_{1} \leq \varepsilon_{1}$. Now, if $a-\delta_{1}>\varepsilon_{1}$ then choose $\varepsilon=\varepsilon_{1}$ and the result is proved. If $a-\delta_{1} \leq \varepsilon_{1}$ then choose $\varepsilon>0$ such that $a-\delta_{1}>\varepsilon$. For any $y=\left(y_{1}, y_{2}, \ldots, y_{N-1}\right) \in Y \backslash\{0\}$ with $y_{1} \leq \varepsilon<\varepsilon_{1}$ it holds that $H_{1}(y)>a-\delta_{1}>\varepsilon$, so in this case the result is proved as well.

Since we established condition $\liminf _{y \rightarrow 0} H_{1}(y) \geq a>0$ in Proposition 6 we can now use $\varepsilon$ from Lemma 3. Define the function $J=\left(J_{1}, \ldots, J_{N-1}\right): Y \rightarrow \mathbb{R}^{N-1}$ such that

$$
J(y)= \begin{cases}H(y) & \text { for } y \in Y \backslash Y_{\varepsilon} \\ H\left(\varepsilon, y_{2}, \ldots, y_{N-1}\right) & \text { for } y \in Y_{\varepsilon}\end{cases}
$$

where $Y_{\varepsilon}=\left\{\left(y_{1}, y_{2}, \ldots, y_{N-1}\right) \in Y: y_{1} \leq \varepsilon\right\}=Y \cap[0, \varepsilon]^{N-1}$. Notice that $J$ is also defined in 0 .

Lemma 4 The function $J$ has the properties: (i) $J$ is continuous. (ii) $J$ takes values in $Y$. (iii) $J$ has no fixed point in $Y_{\varepsilon}$.

Proof. (i) Based on the fact that $H$ is continuous, $J$ is also continuous at points $y$ that are not on the boundary between $Y_{\varepsilon}$ and $Y \backslash Y_{\varepsilon}$. The only non-trivial case is when $y$ is on the boundary between $Y_{\varepsilon}$ and $Y \backslash Y_{\varepsilon}$, that is, in $\left\{\left(y_{1}, y_{2}, \ldots, y_{N-1}\right) \in Y: y_{1}=\varepsilon\right\}$. In this case the limit of $J\left(t^{n}\right)$ for a sequence $\left(t^{n}\right)_{n>1} \subset\left\{\left(y_{1}, y_{2}, \ldots, y_{N-1}\right) \in Y: y_{1}>\varepsilon\right\}$ with $t^{n} \rightarrow y$ should be $J(y)$. Indeed, $J\left(t^{n}\right)=H\left(t^{n}\right) \rightarrow H(y)=H\left(\varepsilon, y_{2}, \ldots, y_{N-1}\right)=J(y)$.

(ii) The fact that $J$ takes values in $Y$ follows from Lemma 1 trivially for the case $\left(y_{1}, y_{2}, \ldots, y_{N-1}\right) \in$ $Y \backslash Y_{\varepsilon}$. For the case $\left(y_{1}, y_{2}, \ldots, y_{N-1}\right) \in Y_{\varepsilon}$ it follows because $\left(\varepsilon, y_{2}, \ldots, y_{N-1}\right) \in Y$ for any $\left(y_{1}, y_{2}, \ldots, y_{N-1}\right) \in Y_{\varepsilon}$, so $J\left(\varepsilon, y_{2}, \ldots, y_{N-1}\right)=H\left(\varepsilon, y_{2}, \ldots, y_{N-1}\right) \in Y$. 
(iii) For an arbitrary $\left(y_{1}, y_{2}, \ldots, y_{N-1}\right) \in Y_{\varepsilon}$ we have $J_{1}\left(y_{1}, y_{2}, \ldots, y_{N-1}\right)=H_{1}\left(\varepsilon, y_{2}, \ldots, y_{N-1}\right)$. Since $y=\left(\varepsilon, y_{2}, \ldots, y_{N-1}\right) \in Y \backslash\{0\}$ with $y_{1} \leq \varepsilon$, by Lemma 3 it holds that $H_{1}\left(\varepsilon, y_{2}, \ldots, y_{N-1}\right)>\varepsilon$. Thus $J_{1}\left(y_{1}, y_{2}, \ldots, y_{N-1}\right)>\varepsilon \geq y_{1}$, so $\left(y_{1}, y_{2}, \ldots, y_{N-1}\right)$ cannot be a fixed point of $J$.

Finally we can establish that the system of equations (16) has a solution. By Lemma 4 the function $J: Y \rightarrow Y$ is continuous. $Y$ is a convex and compact set, so by Brouwer's fixed point theorem $J$ has a fixed point $y^{*}$. The fixed point cannot be in $Y_{\varepsilon}$ by Lemma 4, so $y^{*} \in Y \backslash Y_{\varepsilon}$. Therefore $y^{*}=J\left(y^{*}\right)=H\left(y^{*}\right)$, that is, $y^{*} \in Y \backslash Y_{\varepsilon}$ is a fixed point of $H$. By definition, any fixed point of $H$ is a solution of the system (16). This completes the proof of existence of equilibrium in Theorem 1.

We now prove the part on uniqueness of equilibrium. Setting $N=2$ in equations (16) gives

$$
\begin{aligned}
& x_{0}=G\left(\theta-\theta \int_{0}^{1} \frac{x_{0}-x_{1}}{x_{0}-x_{1}+2 x_{1} u} d u\right) ; \\
& x_{1}=G\left(\theta \int_{0}^{1} \frac{\left(x_{0}-x_{1}\right)(1-2 u)}{x_{0}-x_{1}+2 x_{1} u} d u\right) .
\end{aligned}
$$

Using the notation introduced before, $y_{1}=x_{1} / x_{0} \in(0,1)$, the solution to this system of equations is given by the solution to $H_{1}\left(y_{1}\right)-y_{1}=0$, or

$$
\phi\left(y_{1}\right) \equiv y_{1} G\left(\theta-\theta\left(1-y_{1}\right) I\left(y_{1}\right)\right)-G\left(\theta\left(1-y_{1}\right) J\left(y_{1}\right)\right)=0 .
$$

where

$$
\begin{aligned}
& I\left(y_{1}\right)=\int_{0}^{1} \frac{1}{1-y_{1}+2 y_{1} u} d u=\frac{\log \left(1+y_{1}\right)-\log \left(1-y_{1}\right)}{2 y_{1}} ; \\
& J\left(y_{1}\right)=\int_{0}^{1} \frac{1-2 u}{1-y_{1}+2 y_{1} u} d u=\frac{\log \left(1+y_{1}\right)-\log \left(1-y_{1}\right)-2 y_{1}}{2 y_{1}^{2}} .
\end{aligned}
$$

The derivations above in the proof of Proposition A.1 can readily be used to show that $\lim _{\substack{y_{1} \rightarrow 1 \\ y_{1}>0}} \phi\left(y_{1}\right)=$ $G(\theta)>0, \lim _{\substack{y_{1} \rightarrow 0 \\ y_{1}>0}} \phi\left(y_{1}\right)=0$ and $\lim _{\substack{y_{1} \rightarrow 0 \\ y_{1}>0}} \phi^{\prime}\left(y_{1}\right)<0$. Therefore, if the function $\phi\left(y_{1}\right)$ is strictly convex, the equilibrium is unique. Let us now examine the second derivative of the function $\phi\left(y_{1}\right)$. First we have

$$
\begin{aligned}
\phi^{\prime}\left(y_{1}\right) & =G\left(\theta-\theta\left(1-y_{1}\right) I\left(y_{1}\right)\right)+y_{1} g\left(\theta-\theta\left(1-y_{1}\right) I\left(y_{1}\right)\right) \frac{d\left(-\theta\left(1-y_{1}\right) I\left(y_{1}\right)\right)}{d y_{1}} \\
& -g\left(\theta\left(1-y_{1}\right) J\left(y_{1}\right)\right) \frac{d\left(\theta\left(1-y_{1}\right) J\left(y_{1}\right)\right)}{d y_{1}}
\end{aligned}
$$

and then

$$
\begin{aligned}
\phi^{\prime \prime}\left(y_{1}\right) & =2 g\left(\theta-\theta\left(1-y_{1}\right) I\left(y_{1}\right)\right) \frac{d\left(-\theta\left(1-y_{1}\right) I\left(y_{1}\right)\right)}{d y_{1}}+y_{1} g\left(\theta-\theta\left(1-y_{1}\right) I\left(y_{1}\right)\right) \frac{d^{2}\left(-\theta\left(1-y_{1}\right) I\left(y_{1}\right)\right)}{d y_{1}^{2}} \\
& +y_{1} g^{\prime}\left(\theta-\theta\left(1-y_{1}\right) I\left(y_{1}\right)\right)\left(\frac{d\left(-\theta\left(1-y_{1}\right) I\left(y_{1}\right)\right)}{d y_{1}}\right)^{2}-g^{\prime}\left(\theta\left(1-y_{1}\right) J\left(y_{1}\right)\right)\left(\frac{d\left(\left(1-y_{1}\right) J\left(y_{1}\right)\right)}{d y_{1}}\right)^{2} \\
& -g\left(\theta\left(1-y_{1}\right) J\left(y_{1}\right)\right)\left(\frac{d^{2}\left(\theta\left(1-y_{1}\right) J\left(y_{1}\right)\right)}{d y_{1}^{2}}\right)
\end{aligned}
$$


When $g^{\prime}(\cdot)=0$, this simplifies to

$$
\begin{aligned}
\phi^{\prime \prime}\left(y_{1}\right) & =g\left(\theta-\theta\left(1-y_{1}\right) I\left(y_{1}\right)\right)\left(2 \frac{d\left(-\theta\left(1-y_{1}\right) I\left(y_{1}\right)\right)}{d y_{1}}+y_{1} \frac{d^{2}\left(-\theta\left(1-y_{1}\right) I\left(y_{1}\right)\right)}{d y_{1}^{2}}\right) \\
& -g\left(\theta\left(1-y_{1}\right) J\left(y_{1}\right)\right)\left(\frac{d^{2}\left(\theta\left(1-y_{1}\right) J\left(y_{1}\right)\right)}{d y_{1}^{2}}\right)
\end{aligned}
$$

Notice that

$$
\begin{aligned}
\frac{d\left(-\theta\left(1-y_{1}\right) I\left(y_{1}\right)\right)}{d y_{1}} & =\theta \frac{\log \left[\frac{1+y_{1}}{1-y_{1}}\right]-\frac{2 y_{1}}{1+y_{1}}}{2 y_{1}^{2}} \\
\frac{d^{2}\left(-\theta\left(1-y_{1}\right) I\left(y_{1}\right)\right)}{d y_{1}^{2}} & =-\theta \frac{\log \left[\frac{1+y_{1}}{1-y_{1}}\right]+\frac{2 y_{1}\left(y_{1}^{2}-y_{1}-1\right)}{\left(1+y_{1}\right)^{2}\left(1-y_{1}\right)}}{y_{1}^{3}}
\end{aligned}
$$

So

$$
2 \frac{d\left(-\theta\left(1-y_{1}\right) I\left(y_{1}\right)\right)}{d y_{1}}+y_{1} \frac{d^{2}\left(-\theta\left(1-y_{1}\right) I\left(y_{1}\right)\right)}{d y_{1}^{2}}=\frac{2 \theta}{\left(1-y_{1}\right)\left(1+y_{1}\right)^{2}}>0 \text { for all } y_{1}
$$

Finally

$$
\frac{d\left(-\theta\left(1-y_{1}\right) J\left(y_{1}\right)\right)}{d y_{1}}=\theta \frac{\frac{2 y_{1}\left(2+y_{1}\right)}{1+y_{1}}-\left(2-y_{1}\right) \log \left[\frac{1+y_{1}}{1-y_{1}}\right]}{2 y_{1}^{3}}
$$

So

$$
\frac{d^{2}\left(-\theta\left(1-y_{1}\right) J\left(y_{1}\right)\right)}{d y_{1}^{2}}=\theta \frac{\frac{-2 y_{1}\left(3+2 y_{1}-3 y_{1}^{2}-y_{1}^{3}\right)}{\left(1-y_{1}\right)\left(1+y_{1}\right)^{2}}+\left(3-y_{1}\right) \log \left[\frac{1+y_{1}}{1-y_{1}}\right]}{y_{1}^{4}}<0 \text { for all } y_{1}
$$

Therefore we conclude that $\phi\left(y_{1}\right)$ is strictly convex so the equilibrium is unique. 


\section{References}

[1] Daron Acemoglu and Robert Shimer: "Wage and Technology Dispersion," Review of Economic Studies 67, 585-607, 2000.

[2] Kenneth Burdett and Kenneth L. Judd: "Equilibrium Price Dispersion," Econometrica 51, 955$69,1983$.

[3] Kenneth Burdett and Dale T. Mortensen: "Wage Differentials, Employer Size, and Unemployment," International Economic Review 39, 257-73, 1998.

[4] Chaim Fershtman and Arthur Fishman: "Price Cycles and Booms: Dynamic Search Equilibrium," The American Economic Review 82, 1221-33, 1992.

[5] Han Hong and Matthew Shum: "Using Price Distributions to Estimate Search Costs," RAND Journal of Economics 37, 257-75, 2006.

[6] Maarten C. W. Janssen and José Luis Moraga-González: "Strategic Pricing, Consumer Search and the Number of Firms," Review of Economic Studies 71, 1089-118, 2004.

[7] José Luis Moraga-González and Matthijs R. Wildenbeest: "Maximum Likelihood Estimation of Search Costs," European Economic Review 52, 820-48, 2008.

[8] R. Preston McAfee: "Multiproduct equilibrium price dispersion," Journal of Economic Theory 67, 83-105, 1995. 


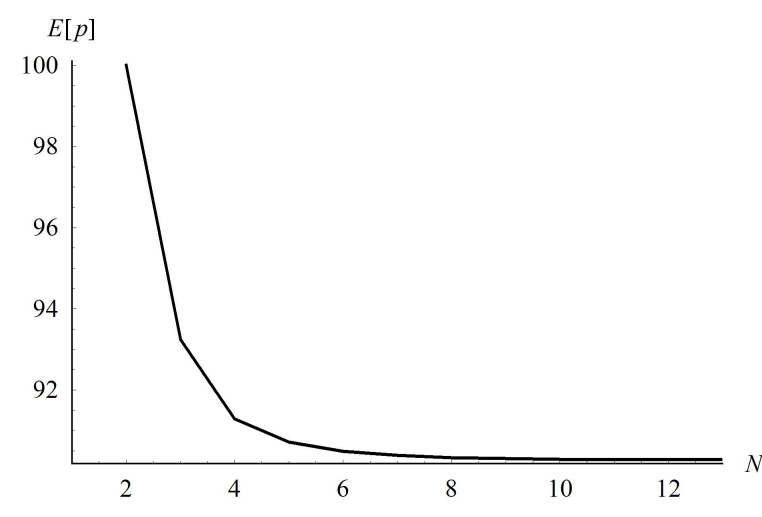

(a) Mean prices

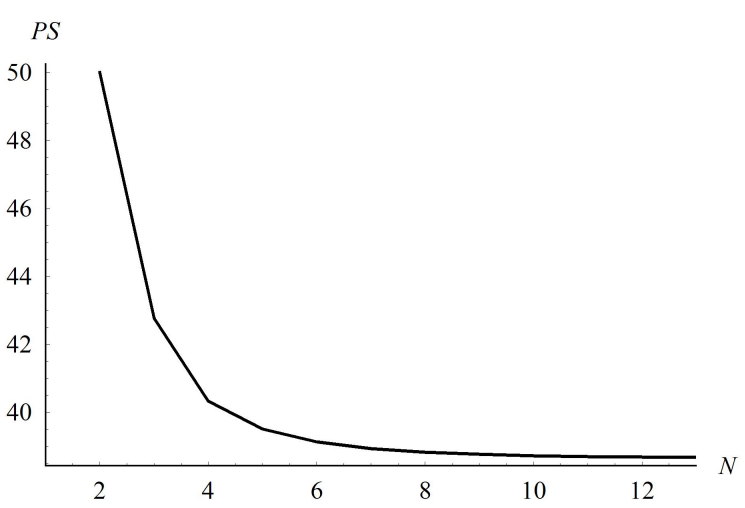

(c) Producer surplus

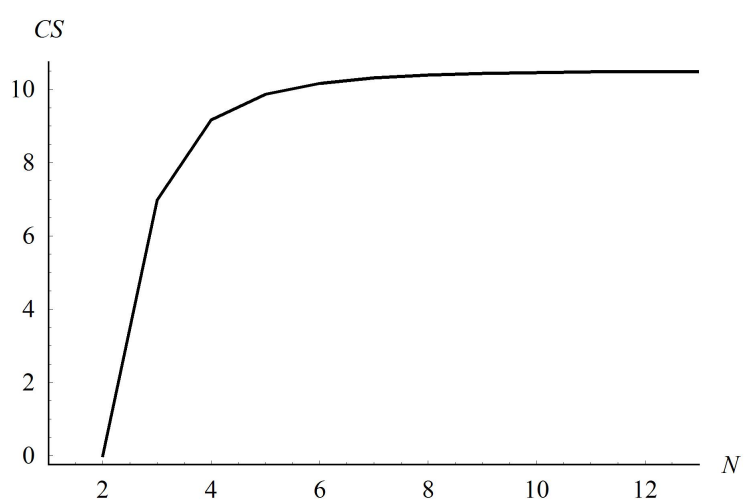

(b) Consumer surplus

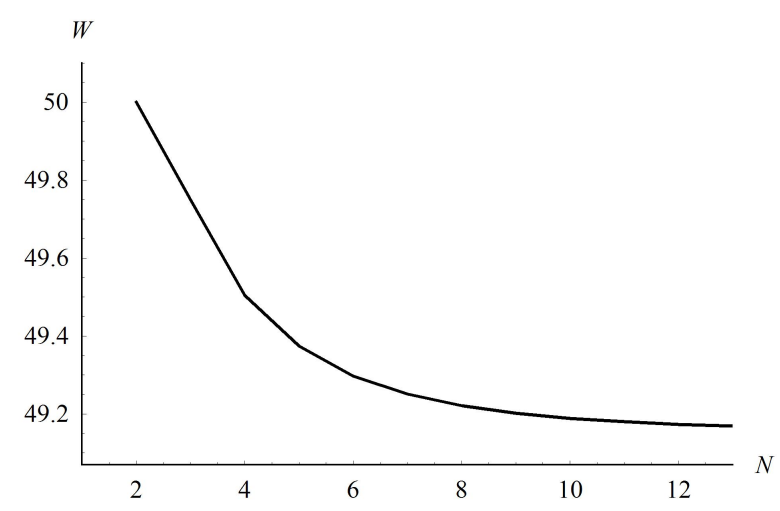

(d) Welfare

Figure 1: Comparative statics of an increase in the number of firms $\left(\left(\nu_{c}, \sigma_{c}\right)=(2.63,1.6)\right)$ 


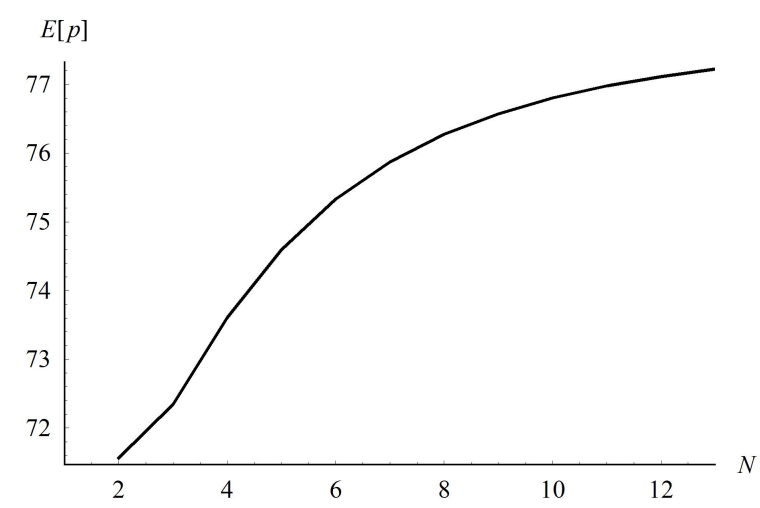

(a) Mean prices

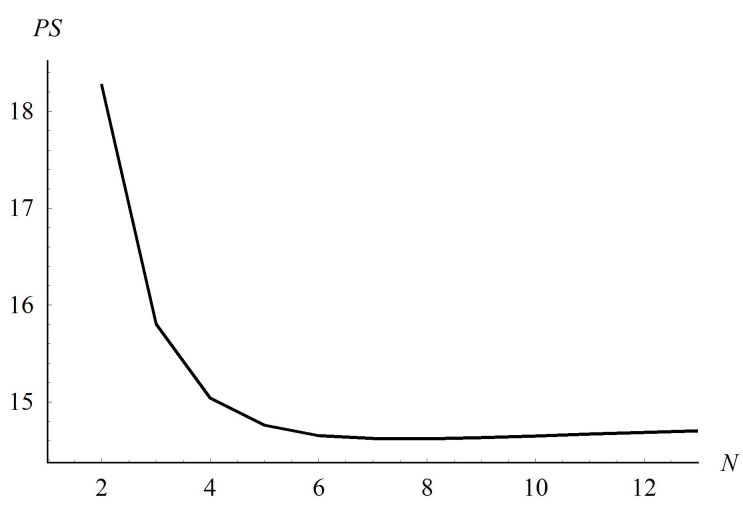

(c) Producer surplus

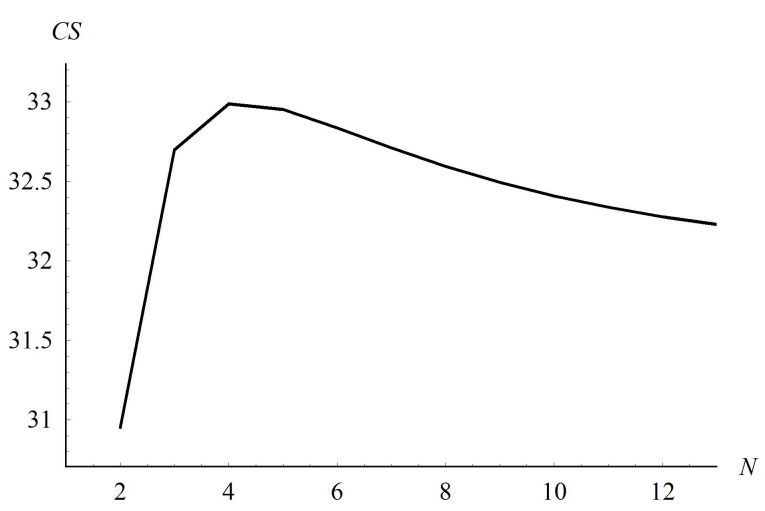

(b) Consumer surplus

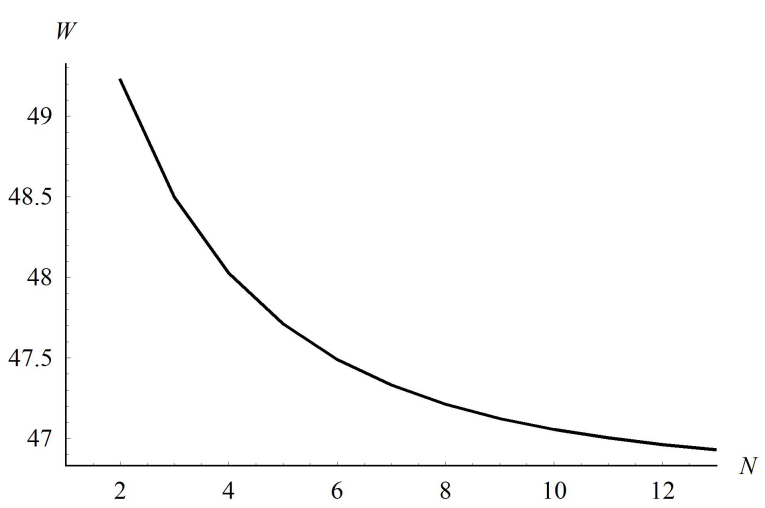

(d) Welfare

Figure 2: Comparative statics of an increase in the number of firms $\left(\nu_{c}=0.79, \sigma_{c}=2.5\right)$ 